\title{
SOME PHOTOMETRIC CHARACTERISTICS OF POPULATION II ORBITAL VISUAL BINARY STARS ${ }^{1}$
}

\author{
A. Bartkevičius ${ }^{2,3}$ and A. Gudas ${ }^{2}$ \\ 2 Institute of Theoretical Physics and Astronomy, Vilnius University, \\ Goštauto 12, Vilnius, LT-01108, Lithuania; \\ 3 Department of Theoretical Physics, Vilnius Pedagogical University, \\ Studentu 39, Vilnius, LT-08106, Lithuania.
}

Received November 20, 2003

\begin{abstract}
A $M_{V}, B-V$ diagram for metal-deficient, kinematically selected thick disk and halo visual binary stars with known orbits is presented. The magnitude differences $\Delta m$ between the components are analyzed.
\end{abstract}

Key words: stars: binaries: visual - stars: fundamental parameters, HR diagram - stars: Population II

\section{INTRODUCTION}

Only orbital binary stars (i.e., binaries with determined orbits) are sources for direct determination of a fundamental parameter stellar mass. The HR diagram of visual binary stars enables us to determine age, helium content and the mixing-length parameter. It also allows us to test evolutionary models. The magnitude differences of the components are used to determine masses of faint components and the mass-luminosity relation.

\section{SELECTION OF STARS}

Population II (thick disk + halo) main-sequence and subgiant orbital visual binary stars are selected from the Bartkevičius \& Gudas catalogs $(2001,2002)$. Stars with Grenon's (1987) kinematic age parameter $f G>0.35$ are also included; here

\footnotetext{
${ }^{1}$ Based on the data from the Hipparcos astrometry satellite (ESA)
} 


$$
f G=1 / 300 \sqrt[2]{U^{2}+2.5 V^{2}+3.5 W^{2}} .
$$

$U$ is the space velocity component directed to the Galactic center, $V$ is the space velocity component directed to the direction of Galactic rotation and $W$ is the space velocity component directed to the North Galactic Pole. The thin disk stars with $[\mathrm{Fe} / \mathrm{H}]<-0.49$ are also included. $[\mathrm{Fe} / \mathrm{H}]$ values are taken from Bartkevičius (1999).

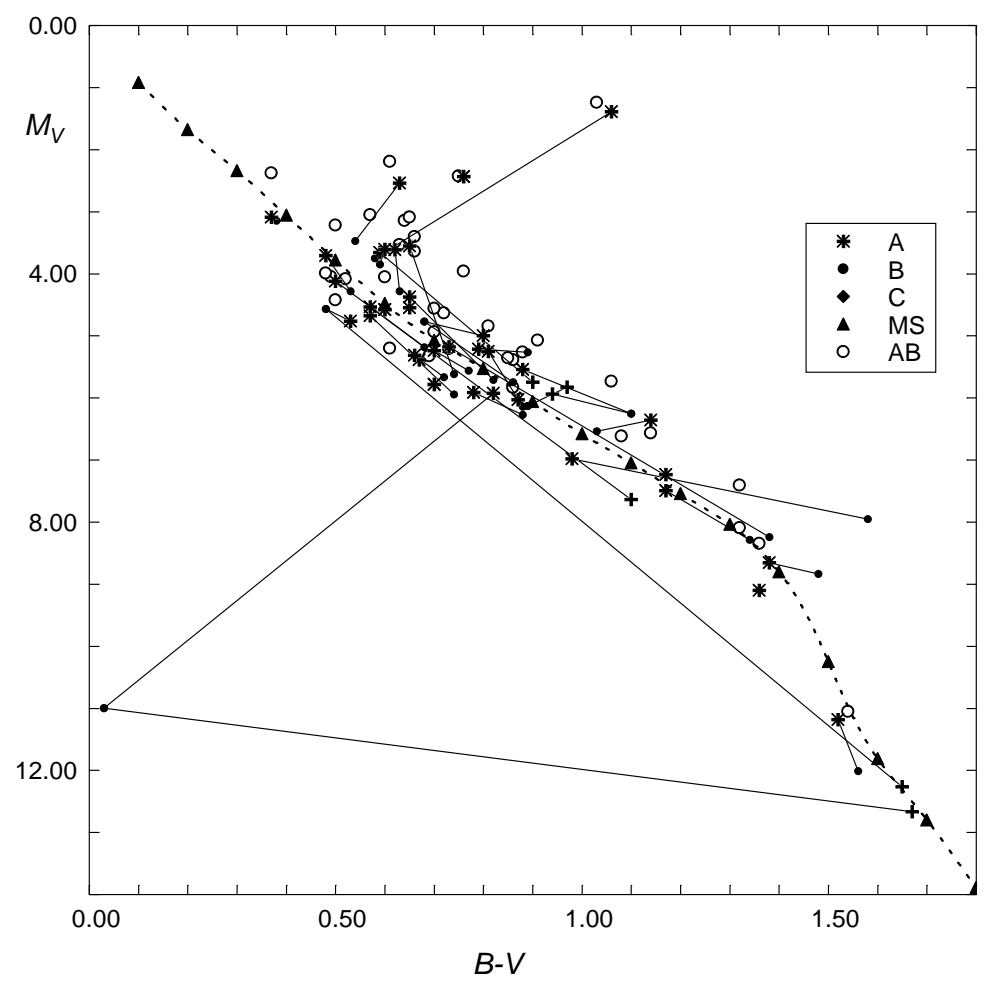

Fig. 1. Color-magnitude diagram for Population II binaries. A,B,C are the resolved components; $\mathrm{AB}$ - combined light of both components; MS - the main-sequence line.

\section{THE DATA}

For parallaxes the same sources are used as in the Bartkevičius \& Gudas $(2001,2002)$ papers. The majority (89\%) of stars have parallaxes with a precision better than $15 \%$. Photometric data are taken from various Hipparcos-Tycho catalogs and the CDS Stellar Data Center. Washington Second Photometric Magnitude Difference Catalog (Mason \& Wycoff 2003) is also consulted for the magnitude differences of the components. 


\section{RESULTS AND CONCLUSIONS}

The $M_{V}, B-V$ diagram for the individual components and the combined light (mostly, $\mathrm{A}+\mathrm{B}$ components) of the selected stars is plotted in Figure 1.

The components of the same system are connected by straight lines. The mean main-sequence line for single stars with a relative parallax error $\sigma_{\pi} / \pi<0.15$ from Hipparcos (ESA 1997) and the Yale Trigonometric Parallaxes Catalogue (van Altena et al. 1995) is shown. A histogram of $V$ magnitude differences between the primary and the secondary components is presented in Figure 2.

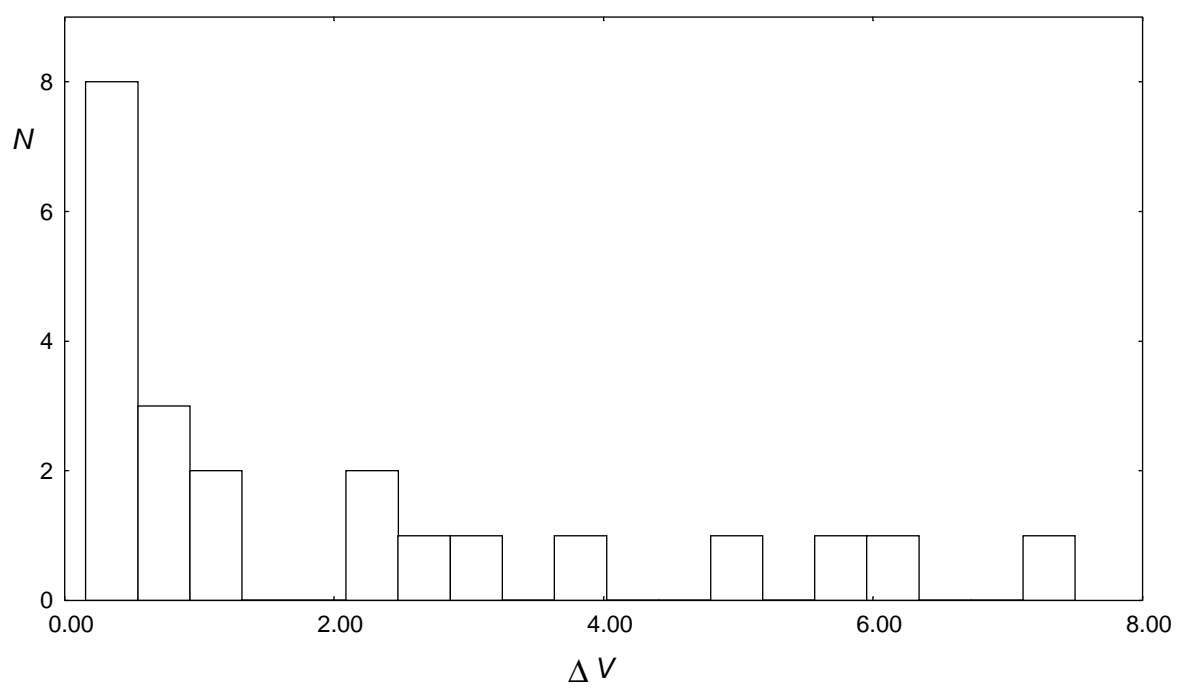

Fig. 2. The histogram of magnitude differences $\Delta V$ of the components.

The region of the color-magnitude diagram from the turn-off point to $B-V=1.0$ is well populated. All the components have color indices $B-V>0.36$, thus the diagram confirms that the binaries in the selected set are relatively old.

As we can see from the $\Delta m$ distribution, more than half of the systems have components with comparable masses. Only several components have large $\Delta m$ values. It would be important to investigate faint low temperature components of these old high-velocity or metal-deficient binaries.

A detailed analysis of color-magnitude diagrams of visual binaries belonging to the halo, thick disk and metal-deficient thin disk will be presented elsewhere. 
ACKNOWLEDGMENTS. In this publication the data from the Strasbourg Stellar Data Center (CDS), the NASA Astronomical Data Center (ADS), and the Washington Double Star Catalog (WDS) maintained by the United States Naval Observatory were used.

\section{REFERENCES}

Bartkevičius A. 1999, Catalog of the Mean [Fe/H] Values of Population II $F-M$ Stars, in preparation

Bartkevičius A., Gudas A. 2001, Baltic Astronomy, 10, 481

Bartkevičius A., Gudas A. 2002, Baltic Astronomy, 11, 153

ESA 1997, The Hipparcos and Tycho Catalogues, SP-1200, ESA

Grenon M. 1987, J. Astrophys. Astron., 8, 123

Mason B. D., Wycoff G. L. 2003, The Second Photometric Magnitude Difference Catalog, U.S. Naval Observatory

van Altena W.F., Lee J. T., Hoffleit E. D. 1995. The General Catalogue of Trigonometric Stellar Parallaxes, 4th edition, Yale University Observatory, CDS Catalog I/238A 